\title{
Biofilm Formation and Susceptibility to Amphotericin B and Fluconazole in Candida albicans
}

\author{
Ali Zarei Mahmoudabadi ${ }^{1,2,}$; Majid Zarrin $^{2}$; Neda Kiasat $^{2}$ \\ ${ }_{1}$ Health Research Institute, Infectious and Tropical Diseases Research Centre, Ahvaz Jundishapur University of Medical Sciences, Ahvaz, IR Iran \\ ${ }^{2}$ Department of Medical Mycology, Ahvaz Jundishapur University of Medical Sciences, Ahvaz, IR Iran \\ ${ }^{*}$ Corresponding author: Ali Zarei Mahmoudabadi, Health Research Institute, Infectious and Tropical Diseases Research Centre, Ahvaz Jundishapur University of Medical Sciences, \\ Ahvaz, IR Iran. Tel: +98-6113330074, Fax:+98-6113332036, E-mail: zarei40@hotmail.com
}

Received: December 25, 2013; Revised: January 21, 2014; Accepted: February 2, 2014

\begin{abstract}
Background: The ability of Candida albicans to form biofilms and adhere to host tissues and biomaterial surfaces is an important factor in its pathogenesis. One of the main characteristics of biofilms is their resistance to broad-spectrum anti-microbial drugs.

Objectives: In the present study the formation of biofilm by C. albicans from different sources was evaluated. In addition, the minimum biofilm inhibitory concentration (MBIC) for two antifungals was evaluated.

Materials and Methods: In total, 120 isolates of C. albicans from different sources (patients with vaginitis, patients with candiduria, bucal cavity and environmental surfaces) were collected. Biofilm formation was determined by the 96 -well micro-titeration plate method. MBIC testing was also performed, using the calorimetric indicator resazurin for amphotericin B and fluconazole.

Results: The results indicated that $100 \%$ of $C$. albicans isolates from different sources had the ability to form biofilms in vitro. Amongst these isolates, $83.3 \%$ of isolates had the maximum potential (4+) to form biofilms, while only one ( $0.9 \%)$ of isolates had the minimum ability (1+) to form biofilms. Our results showed that $65.0 \%$ of the tested isolates are sensitive to amphotericin B at amounts lower than $10 \mu \mathrm{g} / \mathrm{mL}$, while only $26.7 \%$ are sensitive to fluconazole (had MBIC $<10 \mu \mathrm{g} / \mathrm{mL}$ ).

Conclusions: Although biofilm formation was detected in all tested isolates, there were differences in the ability to form biofilms between isolates from different sources. In addition, there were differences in the MBIC against the two examined antifungals, amphotericin B and fluconazole.
\end{abstract}

Keywords: Candida albicans; Amphotericin B; Fluconazole

\section{Background}

Nosocomial infections due to Candida species have increased significantly during the past three decades. Use of a wide range of biomaterial instruments (urinary and in-dwelling vascular catheters, denture appliances, orthopaedic prostheses and heart valves) in clinical practice accelerates infection by Candida species (1-4). In addition, nosocomial Candida infections are more prevalent among immunocompromised individuals and those with a history of diabetes, malignancy, neutropenia, cancer chemotherapy, organ transplantation, hemodialysis, use of broad-spectrum antimicrobial agents and prolonged hospitalization (5-9). Candiduria, vulvovaginal candidiasis and oral candidiasis are the most important forms of the disease.

Candida albicans is still considered as the major etiologic agent in candidiasis and several factors are associated with its pathogenesis. The ability of C. albicans to form biofilms and adhere to host tissues and biomaterial surfaces is an important pathogenesis factor $(5,10,11)$. C. albicans biofilms show a complex three dimensional architecture with extensive spatial heterogeneity and consist of a dense net- work of yeast, hyphae and pseudohyphae encased within a matrix of exopolymeric material (12). Biofilm formation can act as a reservoir of agents, allow co-infection with other pathogens, promote persistence of infection and increase mortality $(13,14)$.

One of the main characteristics of biofilms is their resistance to broad-spectrum anti-microbial drugs (5, $11,15,16)$. Several studies have shown that sessile yeasts (biofilm) are more resistant to amphotericin B, fluconazole, azoles, and echinocandins when compared to planktonic cells $(4,5,11,15)$. Amphotericin B and fluconazole are two important systemic antifungals that are routinely used for the treatment and prophylaxis of systemic candidiasis. However, some isolates of Candida such as, C. glabrata and C. krusei are resistant to fluconazole (17). Decreasing biofilm susceptibility to such antifungals cause an increase in mortality among patients.

\section{Objectives}

The aims of the present study were the evaluation of the formation of biofilm by C. albicans from different resources (such as vagina, urine, mouth and environment), compari-

Implication for health policy/practice/research/medical education:

Although biofilm formation was detected in all tested isolates, there were differences in the ability to form biofilms between isolates from different sources. In addition there were differences in the MBIC against the two examined antifungals, amphotericin B and fluconazole.

Copyright @ 2014, Ahvaz Jundishapur University of Medical Sciences; Published by Kowsar Corp. This is an open-access article distributed under the terms of the Creative Commons Attribution License, which permits unrestricted use, distribution, and reproduction in any medium, provided the original work is properly cited. 
Zarei Mahmoudabadi A et al.

son of biofilms from different resources, and the sensitivity of these biofilms to amphotericin B and fluconazole.

\section{Materials and Methods}

\subsection{Organisms and Identification}

In the present study, 120 isolates of C. albicans, recovered from different sources, were examined. Amongst these, 30 isolates of $C$. albicans were from patients with vulvovaginal candidiasis and 30 from patients with candiduria. Theses 60 isolates were previously collected, identified and preserved in sterile water in our medical mycology laboratory (previous projects). Theses strains were subcultured on Sabouraud dextrose agar (SDA) (Merck, Germany) and re-identified using CHROMagar Candida (CHROMagar Candida Co., France), chlamydoconidia formation on cornmeal agar (Difco, USA) germ tube test and $45^{\circ} \mathrm{C}$ resistance temperature tests (18).

In addition, 230 samples were also collected from mouth, saliva and different environmental surfaces using sterilized swabs. All samples were immediately cultivated on CHROMagar Candida and incubated at $37^{\circ} \mathrm{C}$ for 72 hours. Although, C. albicans on CHROMagar Candida medium were easily identifiable by their light green colonies, all green isolated colonies were confirmed by the tests mentioned above (19). Finally, 60 isolates of C. albicans (30 isolates from buccal cavity and 30 from environmental surfaces) were recovered.

\subsection{Biofilm Formation}

In the present study, biofilm formation was determined using preseterilized polystyrene 96-well microplates (SPL, Korea) (20). For each isolate, a suspension from an overnight culture on SDA was prepared in sterile distilled water and adjusted to $1 \mathrm{McF}$ arland. Each well of the microplate was filled with $180 \mu \mathrm{L}$ of Sabouraud dextrose broth (SDB) (Merck, Germany) supplement with $8 \%$ glucose and then $20 \mu \mathrm{L}$ of the standard suspension of tested isolates was inoculated (21). Microplates were covered with lids and incubated at $35^{\circ} \mathrm{C}$ for 24 hours (20). The medium in wells was removed and washed three times with sterile phosphated buffer solution (PBS). Microplates were stained with Giemsa for 5 minutes and then read at $405 \mathrm{~nm}$ by an Elisa reader $(21,22)$. All tests were done in triplicates and means were calculated. Finally, adherent biofilm layers were scored as either negative; weak $(+)$ (percentage transmittance $(\% \mathrm{~T} \leq$ $20)$ ); moderate, $(++)(\% \mathrm{~T}=20-35) ;$ strong $(+++)(\% \mathrm{~T}=36-50)$ and very strong $(++++)(\% \mathrm{~T} \geq 50)(21)$.

\subsection{Minimum Biofilm Inhibitory Concentration De- tection}

Preparation of antifungals: amphotericin B (Sigma, USA) and fluconazole (Sigma, USA) were prepared at a concentration of $2560 \mu \mathrm{g} / \mathrm{mL}$ in dimethyl sulfoxide (DMSO) and sterile distilled water, respectively (5). Preparation of resazurin: $0.01 \%$ resazurin (Sigma, USA) was prepared in distilled water, mixed to RPMI 1640 (Bio-Idea, Iran) at concentration of $1 / 10$ and sterilized using paper disk filter $(0.22 \mu \mathrm{m})(2)$.

Preparation of the standard suspension: an overnight culture of each tested isolate was prepared on SDA. A suspension of yeast was prepared at $0.9 \% \mathrm{NaCl}$ and adjusted to 3 on the McFarland scale.

Minimum biofilm inhibitory concentration test: the minimum biofilm inhibitory concentration (MBIC) test was performed by a technique using the calorimetric indicator resazurin $(5,13)$. Firstly, $90 \mu \mathrm{L}$ of SDB supplemented with $8 \%$ glucose was added to each well of the microplate. Secondly, $10 \mu \mathrm{L}$ of yeast suspension was also added and incubated at $37^{\circ} \mathrm{C}$ for 48 hours. All media were removed from wells and wells washed three times with $0.9 \% \mathrm{NaCl}$. Serial dilutions of each antifungal agent were prepared from 1280-10 $\mu \mathrm{L} /$ $\mathrm{mL}$ in RPMI 1640. Next, $100 \mu \mathrm{L}$ of each antifungal agent was added to each well and incubated at $37^{\circ} \mathrm{C}$ for 24 hours. A series of wells without antifungal drugs and un-inoculated wells served as positive and negative controls, respectively. All experiments were done in triplicates. When active cells of yeast (live cells) were present in wells, they produced a pinkish color, resorufin from resazurin indicator.

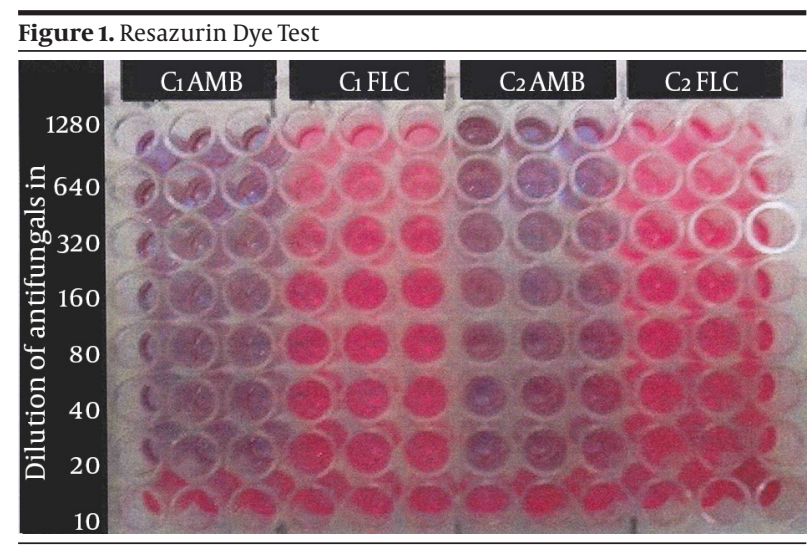

Resazurin dye test for determining MBIC of amphotericine B and fluconazole to C. albicans at serial concentrations from 1280 to $10 \mu \mathrm{L} / \mathrm{mL}, \mathrm{C} 1 \mathrm{AMB}$ C. albicans (No. 1) amphotericine B; C1FLC, C. albicans (No. 1) fluconazole C2AMB, C. albicans (No. 2) amphotericine B; C2FLC, C. albicans (No. 2) fluconazole.

Figure 2. The Frequency of Biofilm Formation in 120 Isolates of C. albicans Recovered From Different Sources

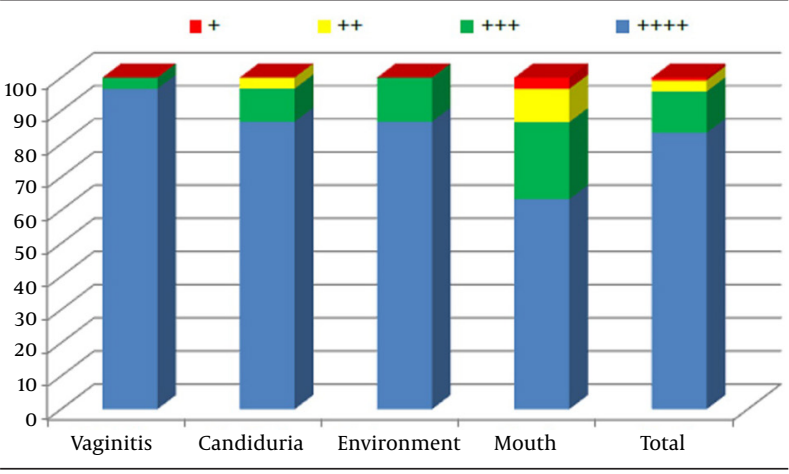

Ability to form biofilm, very high: +4 ; high: $3+$ : moderate: $2+$; low: $1+$ 
Zarei Mahmoudabadi A et al.

Table 1. MBIC of C. albicans From Different Sources Against Different Concentrations of Amphotericin B $(\mu \mathrm{g} / \mathrm{mL})$

\begin{tabular}{lccccc}
\hline $\begin{array}{l}\text { Amphotericin B } \\
\text { Concentrations, } \mu \mathbf{g} / \mathbf{m L} \mathbf{L}\end{array}$ & \multicolumn{2}{c}{ Sources of Tested Candida albicans } & \multicolumn{2}{c}{ Total No. (\%) } \\
\cline { 2 - 5 } & Vaginitis No. (\%) & Candiduria No.(\%) & Environment No. (\%) & Mouth No. (\%) & \\
\hline $\mathbf{1 6 0}$ & $3(10)$ & $0(0.0)$ & $0(0.0)$ & $0(0.0)$ & $3(2.5)$ \\
$\mathbf{1 5 9 - 8 0}$ & $6(20)$ & $0(0.0)$ & $0(0.0)$ & $0(0.0)$ & $6(5.0)$ \\
$\mathbf{7 9 - 4 0}$ & $5(16.7)$ & $0(0.0)$ & $0(0.0)$ & $0(0.0)$ & $5(4.2)$ \\
$\mathbf{3 9 - 2 0}$ & $5(16.7)$ & $8(26.6)$ & $0(0.0)$ & $0(0.0)$ & $13(10.8)$ \\
$\mathbf{1 9 - 1 0}$ & $4(13.3)$ & $11(36.7)$ & $0(0.0)$ & $0(0.0)$ & $15(12.5)$ \\
$<\mathbf{1 0}$ & $7(23.3)$ & $11(36.7)$ & $30(100)$ & $30(100)$ & $78(65.0)$ \\
Total & $30(100)$ & $30(100)$ & $30(100)$ & $30(100)$ & $120(100)$ \\
\hline
\end{tabular}

Table 2. MBIC of C. albicans From Different Sources Against Different Concentrations of Fluconazole, $\mu \mathrm{g} / \mathrm{mL}$

\begin{tabular}{|c|c|c|c|c|c|}
\hline \multirow{2}{*}{$\begin{array}{l}\text { Fluconazole } \\
\text { Concentrations, } \mu \mathrm{g} / \mathrm{mL}\end{array}$} & \multicolumn{4}{|c|}{ Sources of Tested Candida albicans } & \multirow[t]{2}{*}{ Total No. (\%) } \\
\hline & Vaginitis No. (\%) & Candiduria No. (\%) & Environment No.(\%) & Mouth No. (\%) & \\
\hline$>1280$ & $11(36.7)$ & $16(53.3)$ & $12(40.0)$ & $2(6.7)$ & $41(34.2)$ \\
\hline $1280-640$ & $11(36.7)$ & $1(3.3)$ & $17(56.7)$ & $1(3.3)$ & $30(25.0)$ \\
\hline $639-320$ & $6(20.0)$ & $0(0.0)$ & $0(0.0)$ & $0(0.0)$ & $6(5.0)$ \\
\hline $319-160$ & $1(3.3)$ & $0(0.0)$ & $0(0.0)$ & $0(0.0)$ & $1(0.8)$ \\
\hline 159-80 & $1(3.3)$ & $0(0.0)$ & $0(0.0)$ & $1(3.3)$ & $2(1.7)$ \\
\hline 79-40 & $0(0.0)$ & $0(0.0)$ & $0(0.0)$ & $1(3.3)$ & $1(0.8)$ \\
\hline $39-20$ & $0(0.0)$ & $0(0.0)$ & $0(0.0)$ & $1(3.3)$ & $1(0.8)$ \\
\hline 19-10 & $0(0.0)$ & $0(0.0)$ & $0(0.0)$ & $6(20.0)$ & $6(5.0)$ \\
\hline$<10$ & $0(0.0)$ & $13(43.4)$ & $1(3.3)$ & $18(60.0)$ & $32(26.7)$ \\
\hline Total & $30(100)$ & $30(100)$ & $30(100)$ & $30(100)$ & $120(100)$ \\
\hline
\end{tabular}

The lowest concentration of the antifungal drug that maintained the blue color of the calorimetric indicator determined the MBIC (Figure 1).

\section{Results}

\subsection{Biofilm Formation}

In the present study $100 \%$ of the C. albicans isolates that originated from the environment, vagina, urine and mouth had the ability to form a biofilm in vitro. Furthermore, $88.3 \%$ of isolates had the maximum potential to form biofilms (+4), while only one strain $(0.9 \%)$ had the minimum ability to form biofilms $(+1)$. Figure 2 shows the biofilm formation details of 120 tested isolates.

\subsection{MBIC ofC. albicans Against Amphotericin B}

Among the 120 strains of C. albicans tested for MBIC, 65.0\% of the isolates were found to be sensitive to amphotericin $B$ at concentrations lower than $10 \mu \mathrm{g} / \mathrm{mL}$. Our results showed that $30(100 \%)$ of the isolates originating from the environment and mouth had MBIC of $<10 \mu \mathrm{g} / \mathrm{mL}$ (Table 1 ).

\subsection{MBIC of C. albicans to Fluconazole}

Our results indicated that $59.2 \%$ of the tested C. albicans were resistant to fluconazole and had MBIC $>640 \mu \mathrm{g} / \mathrm{mL}$ while only $26.7 \%$ were sensitive to fluconazole and had MBIC $<10 \mu \mathrm{g} / \mathrm{mL}$ (Table 2). Furthermore, 60\% and $43.4 \%$ of mouth and urine isolates had MBIC lower than $<10$ $\mu \mathrm{g} / \mathrm{mL}$, respectively; while, $93.4 \%$ of vaginal isolates and $96.7 \%$ of environmental isolates were resistant to fluconazole at concentrations higher than $320 \mu \mathrm{g} / \mathrm{mL}$.

\section{Discussion}

Candida species are considered as one of the most common human pathogens and the severity of this infection ranges from mild mucocutaneous candidiasis to invasive systemic disease. Moreover, mortality rate of this infection is greater among patients with invasive candidiasis. Several factors contribute to the virulence of Candida species including, production of extracellular enzymes (phospholipase, proteinase, coagulase, esterase and hemolytic activity), biofilm formation and surface adherence $(7,8,23,24)$. In addition, use of several medical biomaterial instruments such as, stents, shunts, implants, endotracheal tubes, pacemakers, orthopaedic prostheses, heart valves and catheters have increased during the last three decades. Use of these instruments has increased the incidence of invasive candidiasis among patients.

C. albicans is an important pathogen in medical device infections because of its ability to form biofilms. It has been shown that the degree of biofilm formation varies 
and correlates with five different switch phenotypes of $C$. albicans (12). Several factors, including host and surface properties, artificial saliva and different environmental conditions affect biofilm formation in C. albicans (2527). In addition, biofilm formation also differed among different species of Candida (28). In our study, similar to other studies, all isolates of $C$. albicans were able to produce biofilms in vitro (28-30). However, the potential to form biofilms varied among C. albicans isolated from different sources. All vaginitis isolates were recovered from patients with vulvovaginal candidiasis and had the maximum potential for biofilm formation $(+4)$ followed by urine, environmental and month strains. Villar-Vidal et al. (28) showed that there is a higher percentage (41.7\%) of biofilm formers among C. albicans recovered from blood samples than oral isolates (31.3\%).

Biofilms in Candida represent a complex of yeast cells, hyphae and pseudohyphae that are encased within a matrix of expolymeric material (12). This complex material is protected from the host immune system and antifungal therapies $(2,11,16)$. One of the most important features of biofilms is their high resistance to antifungal drugs. Tobudic et al. (15) suggested that Candida biofilms show a 1000 -fold greater resistance to antifungals than planktonic cells. Several methods were used for the detection of biofilm susceptibility against antifungal agents, however the resazurin dye test is more popular because of its simplicity (5).

Normally, most strains of C. albicans are sensitive to amphotericin B. In addition, this drug displays good in vitro activity against $C$. albicans biofilms. In a study conducted by Negri et al. (31) only 5.2\% of tested C. albicans from urine, blood and staff hands were resistant to amphotericin B. They also found that $42.1 \%$ of the examined isolates were resistant to fluconazole. On the other hand, all vaginal isolates of Candida in the study of Mohanty et al. (32) were sensitive to fluconazole, while resistance to fluconazole in the study of Richter et al. (33) was 3.7\%. Yang et al. (6) showed that approximately $2.5 \%, 6.5 \%$, and $11.8 \%$ of Candida isolates from middle, north and south regions of Taiwan, respectively, were resistant to fluconazole.

Our study shows that the MBIC for $35 \%$ of all tested isolates was $<10 \mu \mathrm{g} / \mathrm{mL}$ for amphotericin B, however MBIC for all environment and mouth isolates was less than 10 $\mu \mathrm{g} / \mathrm{mL}$. On the other hand, the range of MBIC for pathogenic strains (recovered from urine and vaginitis samples) was variable (Table 1).A considerable high MBIC for vaginitis and urine strains was detected against amphotericin B, respectively. A considerable high MBIC for vaginitis and urine strains was detected against amphotericin B, respectively (11). In the present study, $59.2 \%$ of tested isolates had an MBIC of more than $640 \mu \mathrm{g} / \mathrm{mL}$ for fluconazole and $31.7 \%$ lower than $20 \mu \mathrm{g} / \mathrm{mL}$. Environmental isolates (96.7\%) showed resistance to fluconazole at $>640$ $\mu \mathrm{g} / \mathrm{mL}$. Routine antifungal tests usually detect resistance/ sensitivity to planktonic forms and there are only a few studies that have evaluated antifungals against biofilms.
Our study showed that biofilm formation occurred in all tested isolates of $C$. albicans recovered from different sources. However, the ability to form biofilms was different between isolates. In addition there was different MBIC against the two examined antifungals, amphotericin B and fluconazole.

\section{Acknowledgements}

The authors would like to thank the tropical diseases research center and medical mycology department of Ahvaz Jundishapur University of Medical Sciences for supporting this project. This article was extracted from an MSc thesis by Neda Kiasat.

\section{Authors' Contributions}

Ali Zarei Mahmoudabadi and Majid Zarrin designed and managed the research. Neda Kiasat performed all experiments and analysis of the data. Ali Zarei Mahmoudabadi wrote the draft of the manuscript and edited the final manuscript.

\section{Financial Disclosure}

There was no financial interest.

\section{Funding/Support}

This study was supported by research grant No. OG90135, provided by the Health Research Institute, Infectious and Tropical Diseases Research Centre, Ahvaz Jundishapur University of Medical Sciences, Ahvaz, Iran.

\section{References}

1. Silva S, Negri M, Henriques M, Oliveira R, Williams DW, Azeredo J. Adherence and biofilm formation of non-Candida albicans Candida species. Trends Microbiol. 2011;19(5):241-7.

2. DiDone L, Oga D, Krysan DJ. A novel assay of biofilm antifungal activity reveals that amphotericin B and caspofungin lyse Candida albicans cells in biofilms. Yeast. 2011;28(8):561-8.

3. Hahnel S, Rosentritt M, Burgers R, Handel G, Lang R. Candida albicans biofilm formation on soft denture liners and efficacy of cleaning protocols. Gerodontology. 2012;29(2):e383-91.

4. LaFleur MD, Lucumi E, Napper AD, Diamond SL, Lewis K. Novel high-throughput screen against Candida albicans identifies antifungal potentiators and agents effective against biofilms. $J$ Antimicrob Chemother. 2011;66(4):820-6.

5. Punithavathy PM, Nalina K, Menon T. Antifungal susceptibility testing of Candida tropicalis biofilms against fluconazole using calorimetric indicator resazurin. Indian J Pathol Microbiol. 2012;55(1):72-4.

6. Yang YL, Cheng HH, Ho YA, Hsiao CF, Lo HJ. Fluconazole resistance rate of Candida species from different regions and hospital types in Taiwan. J Microbiol Immunol Infect. 2003;36(3):187-91.

7. Calderone RA, Fonzi WA. Virulence factors of Candida albicans. Trends Microbiol. 2001;9(7):327-35.

8. Gokce G, Cerikcioglu N, Yagci A. Acid proteinase, phospholipase, and biofilm production of Candida species isolated from blood cultures. Mycopathologia. 2007;164(6):265-9.

9. Paul N, Mathai E, Abraham OC, Michael JS, Mathai D. Factors associated with candiduria and related mortality. $J$ Infect. 2007;55(5):450-5.

10. Konopka K, Dorocka-Bobkowska B, Gebremedhin S, Duzgunes N Susceptibility of Candida biofilms to histatin 5 and fluconazole. Antonie Van Leeuwenhoek. 2010;97(4):413-7. 
11. Khan MS, Ahmad I. Antibiofilm activity of certain phytocompounds and their synergy with fluconazole against Candida albicans biofilms. J Antimicrob Chemother. 2012;67(3):618-21.

12. Jin Y, Samaranayake YH, Yip HK, Samaranayake LP. Characterization of switch phenotypes in Candida albicans biofilms. Mycopathologia. 2005;160(3):191-200.

13. Jain N, Kohli R, Cook E, Gialanella P, Chang T, Fries BC. Biofilm formation by and antifungal susceptibility of Candida isolates from urine. Appl Environ Microbiol. 2007;73(6):1697-703.

14. Mohammadi P, Shoaie N, Roudbar Mohammadi S. Isolation and Detection of Yeast Biofilms From Urine Catheters of Infectious Patients. Jundishapur J Microbiol. 2012;5(4):533-6.

15. Tobudic S, Kratzer C, Lassnigg A, Presterl E. Antifungal susceptibility of Candida albicans in biofilms. Mycoses. 2012;55(3):199-204.

16. Tsang CS, Ng H, McMillan AS. Antifungal susceptibility of Candida albicans biofilms on titanium discs with different surface roughness. Clin Oral Investig. 2007;11(4):361-8.

17. Jafari-Nodoushan AA, Kazemi A, Mirzaii F, Dehghani M. Fluconazole Susceptibility Profile of Candida isolates Recovered From Patients Specimens Admitted to Yazd Central Laboratory. Iran J Pharmaceut Res. 2008;7(1):69-75.

18. Ahmadzadeh A, Valavi E, Shamsizadeh A, Zarei Mahmoudabadi A, Hydari M, Ahmadzadeh MA. Fungal urinary tract infection in an infant with posterior urethral valves. Jundishapur J Microbiol. 2011;4(5):.

19. Salehei Z, Seifi Z, Mahmoudabadi AZ. Sensitivity of Vaginal Isolates of Candida to Eight Antifungal Drugs Isolated From Ahvaz, Iran.JundishapurJ Microbiol. 2012;5(4):574-7.

20. Oz Y, Dag I, Kiraz N. Efficacy of disinfectants on Candida biofilms at different concentrations and contact times. British Microbiol Res J. 2012;2(2):40-52.

21. Yigit N, Aktas E, Dagistan S, Ayyildiz A. Investigating Biofilm Production, Coagulase and Hemolytic Activity in Candida Species Isolated From Denture Stomatitis Patients. Eurasian J Med. 2011;43(1):27-32.

22. Paiva LC, Vidigal PG, Donatti L, Svidzinski TI, Consolaro ME. Assessment of in vitro biofilm formation by Candida species isolates from vulvovaginal candidiasis and ultrastructural characteristics. Micron. 2012;43(2-3):497-502.
23. Ghannoum MA. Potential role of phospholipases in virulence and fungal pathogenesis. Clin Microbiol Rev. 2000;13(1):122-43.

24. Samaranayake YH, Dassanayake RS, Jayatilake JA, Cheung BP, Yau JY, Yeung KW, et al. Phospholipase B enzyme expression is not associated with other virulence attributes in Candida albicans isolates from patients with human immunodeficiency virus infection.J Med Microbiol. 2005;54(Pt 6):583-93.

25. Frade JP, Arthington-Skaggs BA. Effect of serum and surface characteristics on Candida albicans biofilm formation. Mycoses. 2011;54(4):e154-62.

26. Silva MP, Chibebe Junior J, Jorjao AL, Machado AK, Oliveira LD, Junqueira JC, et al. Influence of artificial saliva in biofilm formation of Candida albicans in vitro. Braz Oral Res. 2012;26(1):24-8.

27. Thein ZM, Samaranayake YH, Samaranayake LP. In vitro biofilm formation of Candida albicans and non-albicans Candida species under dynamic and anaerobic conditions. Arch Oral Biol. 2007;52(8):761-7.

28. Villar-Vidal M, Marcos-Arias C, Eraso E, Quindos G. Variation in biofilm formation among blood and oral isolates of Candida albicans and Candida dubliniensis. Enferm Infecc Microbiol Clin. 2011;29(9):660-5.

29. Sudjana AN, Carson CF, Carson KC, Riley TV, Hammer KA. Candida albicans adhesion to human epithelial cells and polystyrene and formation of biofilm is reduced by sub-inhibitory Melaleuca alternifolia (tea tree) essential oil. Med Mycol. 2012;50(8):863-70.

30. Lal P, Agarwal V, Pruthi P, Pereira BM, Kural MR, Pruthi V. Biofilm formation by Candida albicans isolated from intrauterine devices. Indian J Microbiol. 2008;48(4):438-44.

31. Negri MF, Faria MG, Guilhermetti E, Alves AA, Paula CR, Svidzinski TIE. Hemolytic activity and production of germ tubes related to pathogenic potential of clinical isolates of Candida albicans. Rev Ciênc Farm Básica. 2010;31(1):89-93.

32. Mohanty S, Xess I, Hasan F, Kapil A, Mittal S, Tolosa JE. Prevalence \& susceptibility to fluconazole of Candida species causing vulvovaginitis. Indian J Med Res. 2007;126(3):216-9.

33. Richter SS, Galask RP, Messer SA, Hollis RJ, Diekema DJ, Pfaller MA. Antifungal susceptibilities of Candida species causing vulvovaginitis and epidemiology of recurrent cases.J Clin Microbiol. 2005;43(5):2155-62. 